\title{
Theoretical and experimental study of physicochemical behavior of binary mixtures: SAFT and PC-SAFT models
}

\author{
BAHMAN SARKOOHAKI ${ }^{\mathrm{a}}$, MOHAMMAD ALMASI ${ }^{\mathrm{b}, *}$ (@) and MEHRNOOSH KARIMKHANI ${ }^{\mathrm{a}}$ \\ ${ }^{a}$ Department of Chemistry, Central Tehran Branch, Islamic Azad University, Tehran, Iran \\ ${ }^{b}$ Department of Applied Chemistry, Faculty of Science, Malayer University, Malayer 65174, Iran \\ E-mail: m.almasi@malayeru.ac.ir
}

MS received 12 August 2018; revised 4 April 2019; accepted 11 April 2019; published online 20 June 2019

\begin{abstract}
The type and magnitude of interactions between diisobutyl ketone (DIBK) and a homologous series of 2-alkanols (2-propanol up to 2-pentanol) are studied in this paper using density and viscosity data. Studies have been carried out in a wide range of temperature (293.15 K to $323.15 \mathrm{~K})$, atmospheric pressure, and in the entire range of composition. The calculated excess molar volumes of the above-mentioned systems are positive, and the viscosity deviations are negative. For the correlation of the density values of binary systems, the statistical association fluid theory (SAFT) and perturbed chain statistical association fluid theory (PC-SAFT) have been used, and the output of two models are compared by average absolute deviation. The results show that the PC-SAFT model is more efficient. Densities and viscosities reported for binary systems along with applied models are all novels, and so far no reports have been investigated in the scientific papers.
\end{abstract}

Keywords. DIBK; density; viscosity; models.

\section{Introduction}

Diisobutyl ketone (DIBK) is a low cast polar compound ( $\mu=2.70 \mathrm{D}$ and $\varepsilon=9.9$ at $20^{\circ} \mathrm{C}$ ) that often is used as a solvent for various coating systems especially as paint removers, acrylic coatings, and cleaning solutions. Furthermore, DIBK is applied as an extraction solvent in the transparent paper industry and production of synthetic leathers. It is a colorless and stable liquid with the high boiling point and slow evaporating property that has limited water solubility but is miscible with alcohols. Excellent viscosity reduction in the high solid's coatings and non-HAP (Hazardous Air Pollutant) solvent are the other advantages of this chemical. ${ }^{1}$ Our studies are directed to investigate non-aqueous binary mixtures by physicochemical properties ${ }^{2,3}$ to provide useful information about the molecular interactions. The density, viscosity and some other properties of the DIBK and 2alkanol binary systems are reported here. It is expected that due to the presence of active sites for hydrogen bonding in both the compounds, the macroscopic interactions of their mixtures could be explained using this molecular property. Therefore, this study was conducted to understand the intermolecular interactions especially, the possibility of forming the hydrogen bond in the mixtures between the unlike components DIBK and 2alkanol with differing hydrocarbon chain length. Binary mixtures involving DIBK ketone, have many applications in the fields of paints, printing inks, and extraction technology. To our knowledge, this is the first reported density and viscosity for the binary mixtures mentioned in the literature.

In the recent years, the development and application of theoretical models in a wide range of temperature and pressure are extended. One approach that has grown popular due to its ability to predict the thermodynamic properties of alcohol containing solutions is the SAFT model. Recently, a more common version of this model has been presented by Gross and Sadowski, called PCSAFT. Density values for binary systems have been correlated with the SAFT model. Intending to establish a comparison, the PC-SAFT model has also been used. The output results show that the data of PC-SAFT model are more accurate.

\footnotetext{
*For correspondence

Electronic supplementary material: The online version of this article (https://doi.org/10.1007/s12039-019-1630-9) contains supplementary material, which is available to authorized users.
} 
Table 1. Densities, $\rho$, and viscosities, $\eta$, of pure components at various temperatures and $P=0.1 \mathrm{MPa}$.

\begin{tabular}{|c|c|c|c|c|c|}
\hline \multirow[b]{2}{*}{ Chemical Name } & \multirow[b]{2}{*}{$T / \mathrm{K}$} & \multicolumn{2}{|r|}{$\rho\left(\mathrm{g} \cdot \mathrm{cm}^{-3}\right)$} & \multicolumn{2}{|c|}{$\eta(\mathrm{mPa} \cdot \mathrm{s})$} \\
\hline & & Expt. & Lit. & Expt. & Lit. \\
\hline \multirow[t]{7}{*}{ DIBK } & 293.15 & 0.8058 & $0.80530^{4} 0.80600^{5} 0.8069^{6}$ & 0.95 & $0.903^{5}$ \\
\hline & 298.15 & 0.8016 & $0.8022^{6}$ & 0.905 & \\
\hline & 303.15 & 0.7973 & & 0.86 & $0.765^{5}$ \\
\hline & 308.15 & 0.7928 & & 0.81 & \\
\hline & 313.15 & 0.7886 & $0.78995^{5}$ & 0.76 & $0.665^{5}$ \\
\hline & 318.15 & 0.7844 & & 0.72 & \\
\hline & 323.15 & 0.7808 & & 0.67 & \\
\hline \multirow[t]{7}{*}{ 2-Propanol } & 293.15 & 0.7854 & $0.78535^{7}$ & 2.42 & $2.414^{7}$ \\
\hline & 298.15 & 0.7811 & $0.78110^{7}$ & 2.08 & $2.070^{7}$ \\
\hline & 303.15 & 0.7768 & $0.77712^{7}$ & 1.80 & $1.785^{7}$ \\
\hline & 308.15 & 0.7724 & $0.77232^{7}$ & 1.56 & $1.546^{7}$ \\
\hline & 313.15 & 0.7680 & $0.76879^{7}$ & 1.36 & $1.347^{7}$ \\
\hline & 318.15 & 0.7634 & $0.76397^{7}$ & 1.19 & $1.76^{7}$ \\
\hline & 323.15 & 0.7588 & & 1.05 & $1.033^{7}$ \\
\hline \multirow[t]{7}{*}{ 2-Butanol } & 293.15 & 0.8067 & & 3.67 & \\
\hline & 298.15 & 0.8027 & $0.802682^{8}$ & 3.04 & $3.074^{8}$ \\
\hline & 303.15 & 0.7984 & $0.798374^{8}$ & 2.54 & $2.552^{8}$ \\
\hline & 308.15 & 0.7941 & $0.793881^{8}$ & 2.13 & $2.144^{8}$ \\
\hline & 313.15 & 0.7898 & $0.789487^{8}$ & 1.80 & $1.818^{8}$ \\
\hline & 318.15 & 0.7852 & $0.784988^{8}$ & 1.54 & $1.558^{8}$ \\
\hline & 323.15 & 0.7806 & & 1.33 & \\
\hline \multirow[t]{7}{*}{ 2-Pentanol } & 293.15 & 0.8093 & & 3.97 & \\
\hline & 298.15 & 0.8053 & $0.8055^{9}$ & 3.32 & $3.45^{9}$ \\
\hline & 303.15 & 0.8012 & $0.8010^{9}$ & 2.81 & $2.77^{9}$ \\
\hline & 308.15 & 0.7970 & $0.7969^{9}$ & 2.37 & $2.32^{9}$ \\
\hline & 313.15 & 0.7927 & $0.7926^{9}$ & 1.99 & $1.92^{9}$ \\
\hline & 318.15 & 0.7884 & & 1.66 & \\
\hline & 323.15 & 0.7840 & & 1.39 & \\
\hline
\end{tabular}

\section{Experimental}

\subsection{Materials}

The materials used in this paper (DIBK, 2-propanol, 2-butanol and 2-pentanol) are from Merck with the purity higher than $99 \%$ and used without further purifications. In Table 1, values of the density and the viscosity of the pure materials are presented along with the values reported in the scientific literature. ${ }^{4-9}$ The difference between experimental density and viscosity, and reported values in literature could be due to the type and amount of impurities, variety in the sources of materials (such as and Merck, Sigma, Fluka and...) and the differences in measurement techniques.

\subsection{Measurements}

A fully automatic apparatus namely SVM 3000 Anton-Paar Stabinger viscometer has been used to measure the density and viscosity. The device, which operates by the modified Couette principle, contains a rapid rotating outer tube and an inner measuring bob which rotates more slowly. Because the density and viscosity values are very sensitive to temperature, they are controlled by a built-in thermoelectric thermostat. Every day and before each series of measurements, the apparatus is calibrated using doubly distilled water and dry air. To measure the density and viscosity, the mixtures are provided precisely before use by mass on an analytical balance (Mettler AE 163, Switzerland) with the precision of \pm 0.01 $\mathrm{mg}$. At first, the liquid with low volatility is prepared, and a maximum of cautions are taken to prevent evaporation of the samples. To provide each mixture, ten compositions are prepared and their physical properties in the range of mole fraction were measured. For density measurements, the uncertainty was $1 \times 10^{-3} \mathrm{~g} \cdot \mathrm{cm}^{-3}$ and for viscosity measurements, the relative uncertainty is 0.02 . The uncertainty in the mole fraction is estimated at $\pm 1 \times 10^{-3}$.

\section{Results and Discussion}

\subsection{Densities and excess molar volumes}

Table S1 (Supplementary Information) shows the density and viscosity values for the pure materials and 
binary mixtures. The excess molar volume $V_{m}^{E}$ was calculated as follows:

$V_{m}^{E}=\sum_{i=1}^{N} x_{i} M_{i}\left(\rho^{-1}-\rho_{i}^{-1}\right)$

where $\rho_{i}$ is the density of pure component $i, \rho$ is the density of the mixture, $M_{i}$ is the molar mass, $x_{i}$ is the mole fraction, and $N$ represents the number of components in the mixture. Generally, $V_{m}^{E}$ for binary systems is correlated by the Redlich-Kister ${ }^{10}$ equation

$X_{m}^{E}=x_{1}\left(1-x_{1}\right) \sum_{k=0}^{N} A_{k}\left(1-2 x_{1}\right)^{k}$

where $X_{m}^{E}$ stands for $V_{m}^{E}$ or $\Delta \eta, A_{k}$ represents the adjustable coefficients and $x_{1}$ is the mole fraction of diisobutyl ketone. Values of $V_{m}^{E} \Delta \eta$ and $A_{k}$ have been reported in Tables S2 and S3 (Supplementary Information). The standard deviation is calculated as follows:

$\left.\sigma=\left[\sum X_{\exp }^{E}-X_{\text {calc }}^{E}\right)^{2} /(n-p)\right]^{1 / 2}$

$X_{\text {exp }}^{E}$ are experimental data and $X_{\text {calc }}^{E}$, the calculated values. Standard deviations are reported in Table S3 (Supplementary Information).

Figure 1 shows the graph of $V_{m}^{E}$ for binary mixtures at $T=298.15 \mathrm{~K}$. As illustrated in this diagram, the excess molar volumes for all systems are positive over the entire range of concentration. In general, for the interpretation of $V_{m}^{E}$ values, three different effects should be considered. Physical interactions, due to dispersion forces and other weak interactions, make a positive contribution to the excess molar volumes. Chemical interactions involve the formation of strong bonds such as hydrogen bonds and charge transfer complexes. Such interactions have a negative effect on $V_{m}^{E}$. Structural effects caused by the fitting of one component inside the structure of other molecules have both negative and positive effects on the excess molar volumes. ${ }^{11}$ The interactions occurring in the mixing of DIBK (1) + 2-alkanol can be divided into two major categories: i) Favorable interactions between $\mathrm{C}=\mathrm{O}$ of ketone and $-\mathrm{OH}$ of alcohol which have the negative contribution on $V_{m}^{E}$. ii) Unfavourable interactions between polar head groups and apolar substituent. The sign $V_{m}^{E}$ is determined by the outcomes of two opposite effects.

The positive deviation of $V_{m}^{E}$ for DIBK + 2-alkanol system shows that the second effect is more dominant and steric hindrances of molecules along with unfavorable interactions governed the behavior of the mixture. By increasing the chain length of alcohols (from 2propanol up to 2-pentanol) values of excess molar volumes increased. This behavior may be attributed to

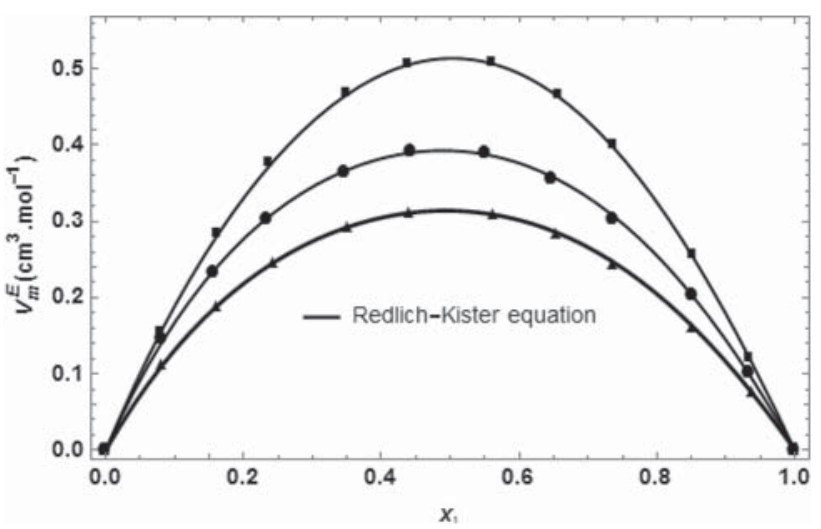

Figure 1. Excess molar volumes $V_{m}^{E}$ vs. mole fraction of DIBK for binary mixtures of DIBK with ( $\mathbf{\Delta})$ 2-propanol, $(\bullet)$ 2-butanol, (ם) 2-pentanol at $T=298.15 \mathrm{~K}$.

the decrease in the polar characteristics of mixtures which resist the formation of intermolecular interactions and the steric hindrances created by the lengthening of the alcohols carbon chain. Obviously, $V_{m}^{E}$ values for all binary systems increase with a rise in the temperature because it leads to an increase in the intermolecular distance and the formation of solutions with less order.

\subsection{Dynamic viscosities}

The following equation was used to calculate the viscosity deviation

$\Delta \eta=\eta-x_{1} \eta_{1}-x_{2} \eta_{2}$

In the above equation, $\eta$ shows the mixture viscosity and $\eta_{i}$ is the viscosity of the pure components. Viscosity deviations for all binary systems at $T=298.15 \mathrm{~K}$ are shown in Figure 2. As is evident from this figure, the rise in the chain length of the alcohols makes an increase in the viscosity deviations which can be attributed to the weakening of the interactions in solutions with high chain alcohol. $\Delta \eta$ values were correlated by the Redlich - Kister equation, and adjustable coefficients are reported in Table S3 (Supplementary Information) along with standard deviations.

\subsection{SAFT model}

The assumption of this model ${ }^{12,13}$ is that the liquid is made from equal-sized hard spheres and interaction between these spheres is calculated by addition of a dispersive repulsive potential. Then, to form a chain, two or more sticky spots are assigned to each sphere. In the final step, in certain places of the chain, specific sites are considered for interaction. The existence of these sites enabling the liquid to associate through attractive interactions such as hydrogen bonds. Each of 


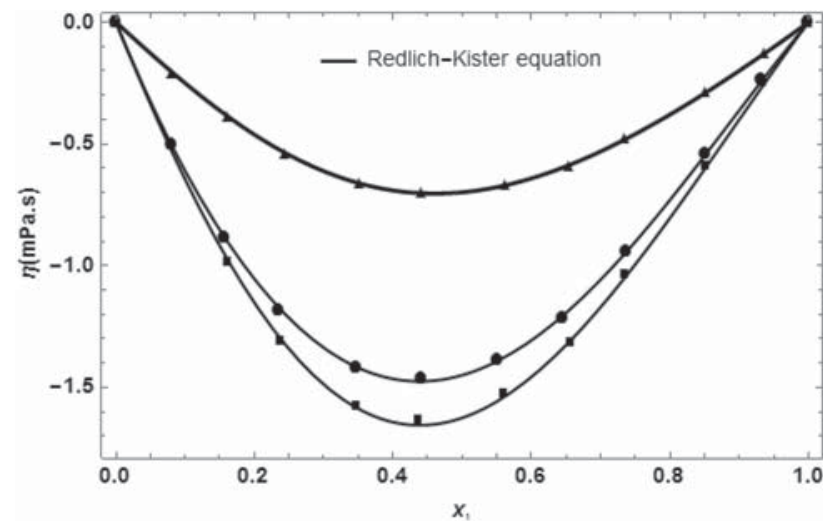

Figure 2. Viscosity deviations $\Delta \eta$ vs. mole fraction of

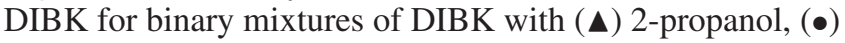
2-butanol, (ם) 2-pentanol at $T=298.15 \mathrm{~K}$.

the mentioned steps is described regarding the residual Helmholtz energy

$a^{\text {res }}=a^{\text {seg }}+a^{\text {chain }}+a^{\text {assoc }}$

$a^{\text {seg }}$ contain the hard sphere and dispersion parts. Sum of chain-forming bonds among the segments are shown by $a^{\text {chain }}$, and the association energy of the liquid by $a^{a s s o c}$.

\section{$3.4 \quad$ PC-SAFT model}

This model ${ }^{14,15}$ is more complete than SAFT, and based on the residual Helmholtz energy is shown as follows:

$a^{r e s}=a^{h c}+a^{d i s}+a^{a s s o c}$

$a^{d i s}$ is the contribution of dispersion interaction and $a^{h c}$ is the hard-sphere contribution. SAFT and PC-SAFT models are presented elsewhere in more details and not provided here. We used both equations to correlate the density of pure components and binary mixtures. At first, the pure parameters for the two models should be calculated. These parameters are obtained by fitting of pure densities and vapor pressures at various temperatures. For the associated components, five parameters and for nonassociated materials, three parameters are needed. Mentioned parameters for the pure state are reported in our previous paper and not presented here. Then, the pressure of the mixture is calculated for two different models by equations 5 to 6 . The final equation in both models contains an adjustable parameter namely $k_{i j}$, which corrects the dispersion forces in the mixtures. Values of this parameter were obtained by iteration method and when the differences between the experimental pressure and SAFT or PC-SAFT values were minimum. The proposed method was used for two models and accordingly, values of $k_{i j}$ (generally independent of temperature) were obtained for SAFT or PC-SAFT

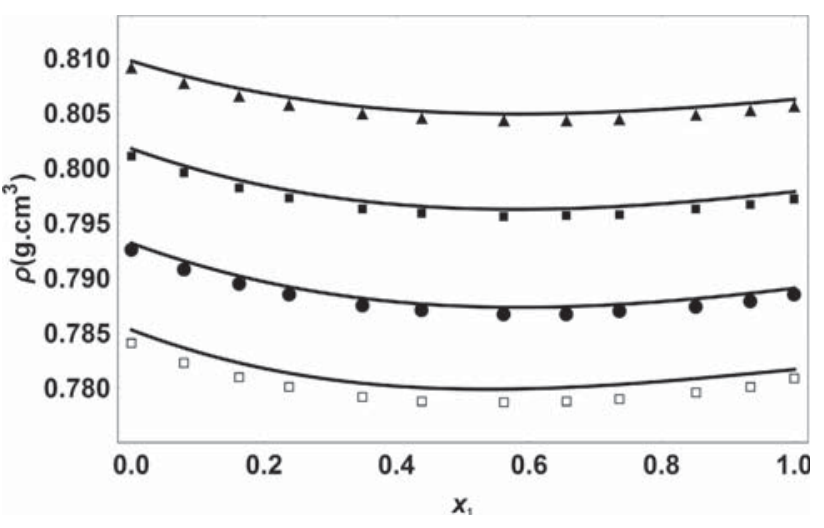

Figure 3. Experimental and calculated values of density for

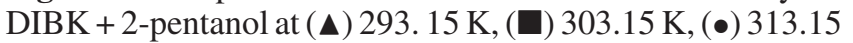
K, ( $\square) 323.15$ K. (-) SAFT model.

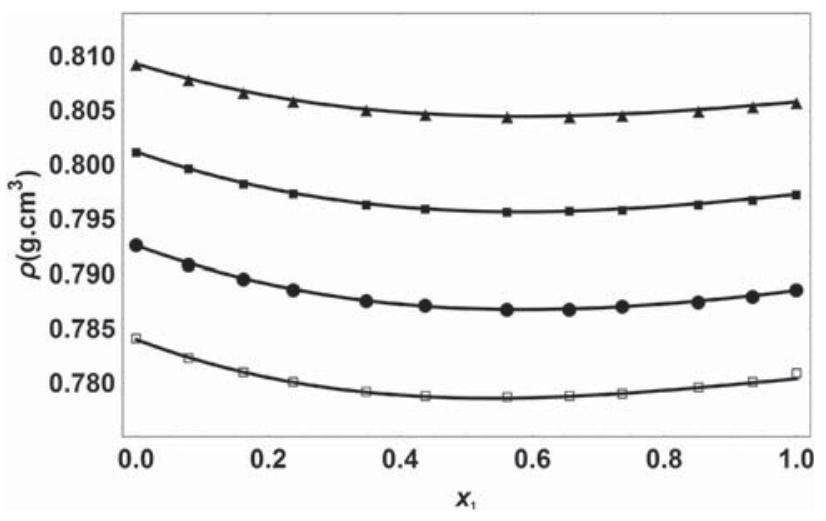

Figure 4. Experimental and calculated values of density DIBK + 2-pentanol at ( $\mathbf{\Delta}) 293.15 \mathrm{~K},(\boldsymbol{\square}) 303.15 \mathrm{~K},(\bullet) 313.15$ K, ( $\square$ ) 323.15 K. (-) PC-SAFT model.

models and reported in Table S4 (Supplementary Information) along with the AAD values. These data reveal that two models yielded small average absolute deviation (AAD) and provided good correlations, but the PC-SAFT model provides more accurate results. Maximum AAD for SAFT model is $2.15 \%$ and for PC-SAFT $1.18 \%$. In fact, the better outcome of PC-SAFT may be due to the intrinsic assumptions of this theory, and generally, in most cases, the PC-SAFT model has shown better results for associated mixtures. Figures 3 and 4 show the comparisons of experimental and calculated densities by SAFT and PC-SAFT models for DIBK + 2-pentanol mixture.

\section{Conclusions}

New density and viscosity data for binary mixtures of DIBK and short chain alcohols (2-propanol up to 2pentanol) are presented in this paper. The excess and 
deviation quantities were calculated for the above systems and described based on the type and nature of interactions in the mixtures. The general structure of binary mixtures is governed by steric hindrance of the nonpolar bulky chain. Two models SAFT and PCSAFT have been used to describe the thermodynamic behaviour of binary systems, and based on the AAD values; the PC-SAFT model has better results for mentioned solutions.

\section{Supplementary Information (SI)}

Supplementary data associated with this article can be found at www.ias.ac.in/chemsci.

\section{References}

1. Papa J 2005 Ketones In Ullmann's Encyclopedia of Industrial Chemistry (Weinheim: Wiley-VCH)

2. Almasi M and Daneshi R 2018 Investigation of Molecular Interactions in Binary Mixtures of n-Butyl Acetate and (C6 - C10) 1-Alkanol: PC-SAFT Model J. Chem. Eng. Data 633881

3. Moftakhar M and Almasi M 2017 Studies on physicochemical behavior of binary mixtures containing propanal and Alkan-2-ol J. Chem. Thermodyn. 113315

4. Ozmen D 2006 (Liquid + liquid) equilibria of (water + propionic acid + methyl isoamyl ketone or diisobutyl ketone or ethyl isoamyl ketone) at $\mathrm{T}=298.2 \mathrm{~K}$ Fluid Phase Equilib. 25070

5. Stroscs F H, Gablea M and Rounds G C 1947 The Vapor Pressures and Some Other Properties of Di-\&butyl Ketone and Di-ibutylcarbinol J. Am. Chem. Soc. 691629
6. Sellers P 1970 Enthalpies of Formation of Some Aliphatic Branched Ketones J. Chem. Thermodyn. 2211

7. Pang F, Seng C, Teng T and Ibrahim M H 2007 Densities and viscosities of aqueous solutions of 1-propanol and 2-propanol at temperatures from $293.15 \mathrm{~K}$ to $333.15 \mathrm{~K}$ J. Mol. Liq. 13671

8. Spasojevic V D, Djordjevic B D, Serbanovic S P, Radovic I R and Kijevcanin M L 2014 Densities, Refractive Indices, Viscosities, and Spectroscopic Study of 1-Amino-2-propanol + 1-Butanol and + 2-Butanol Solutions at (288.15 to 333.15) K J. Chem. Eng. Data 59 1817

9. Riggio R, Martinez H E and Solimo H N 1986 Densities, Viscosities, and Refractive Indexes for the Methyl Isobutyl Ketone + Pentanols Systems, Measurements and Correlations J. Chem. Eng. Data 31235

10. Redlich O J and Kister A T 1948 Algebraic representation of thermodynamic properties and the classification of solutions Ind. Eng. Chem. 40345

11. Lomte S B, Bawa M J, Lande M K and Arbad B R 2009 Densities and Viscosities of Binary Liquid Mixtures of 2Butanone with Branched Alcohols at (293.15 to 313.15) K J. Chem. Eng. Data 54127

12. Huang S H and Radosz M 1993 Equation of state for small, large, Polydisperse and associating molecules: extension to fluid mixtures Ind. Eng. Chem. Res. 32 762

13. Huang S H and Radosz M 1990 equation of state for small, large, Polydisperse and associating molecules Ind. Eng. Chem. Res. 292284

14. Gross J and Sadowski G 2001 Perturbed-Chain SAFT. An equation of state based on a perturbation theory for chain molecules Ind. Eng. Chem. Res. 401244

15. Gross J and Sadowski G 2002 Application of the perturbed-chain SAFT equation of state to associating systems Ind. Eng. Chem. Res. 415510 